\title{
Synthesis and Characterization of the Superconductor $\mathrm{Mo}_{3} \mathrm{Sb}_{7}$
}

\author{
V.H. TRAN \\ W. Trzebiatowski Institute of Low Temperature and Structure Research \\ Polish Academy of Sciences, P.O. Box 1410, 50-950 Wrocław, Poland \\ AND Z. BukOWskI \\ Laboratory for Solid State Physics, ETH Zürich, 8093 Zürich, Switzerland \\ We synthesized a polycrystalline sample of the superconductor \\ $\mathrm{Mo}_{3} \mathrm{Sb}_{7}$ and characterized its basic properties by measurements of magne- \\ tization, electrical resistivity and specific heat. The measurements establish \\ a superconducting phase transition at $T_{\mathrm{c}}=2.25(0.05) \mathrm{K}$. In applied mag- \\ netic fields, the magnetization and resistivity show the behavior of type-II \\ superconductor. From the specific heat measurement, the electronic specific \\ heat coefficient, $\gamma$, specific heat jump at $T_{\mathrm{c}}, \Delta C\left(T_{\mathrm{c}}\right) / \gamma T_{\mathrm{c}}$, and the Debye \\ temperature $\Theta_{\mathrm{D}}$, are evaluated to be $34.5 \mathrm{~mJ} /\left(\mathrm{mol} \mathrm{K}^{2}\right), 1.56$ and $283 \mathrm{~K}$, \\ respectively. From analysis of the specific heat data we suggest that this \\ compound belongs to an intermediate strong coupling regime in the BCS \\ context. The observed enhancement in the $\gamma$-value suggests a substantial \\ contribution of Mo- $4 d$-electrons to the density of states. Our experimental \\ data do not support interpretation of superconductivity in $\mathrm{Mo}_{3} \mathrm{Sb}_{7}$ in terms \\ of neither unconventional superconductivity or spin fluctuations proposed \\ previously.
}

PACS numbers: 74.25.Bt, 74.70.--b

\section{Introduction}

The intermetallic $\mathrm{Mo}_{3} \mathrm{Sb}_{7}$ compound was reported to be a superconductor below the critical temperature of $2.08 \mathrm{~K}$ [1]. This interesting property has been documented on the basis of magnetic susceptibility, electrical resistivity [1] and point-contact (PC) Andreev-reflection [2] experiments on a single crystal. Up to now, there have been several studies pointing towards the nature of the pairing mechanism. Based on PC Andreev-reflection data Dmitriev et al. [3] have suggested a $(s+g)$-wave or another unconventional superconductivity. On the second hand, Candolfi et al. [4] have reported magnetic susceptibility, specific heat, and 
electrical resistivity down to $0.6 \mathrm{~K}$ and have interpreted their data within the framework of the spin fluctuation theory. Thus, the issue of pairing mechanism becomes debated and the proper superconductivity nature of $\mathrm{Mo}_{3} \mathrm{Sb}_{7}$ deserves studies in more detail. Therefore, we undertaken synthesis of a polycrystalline sample of $\mathrm{Mo}_{3} \mathrm{Sb}_{7}$ and in this work we report on physical characterization by means of magnetic susceptibility, magnetization, electrical resistivity and specific heat measurements. The experimental data indicate the presence of the superconducting state below $2.25 \pm 0.05 \mathrm{~K}$, i.e., of $0.2 \mathrm{~K}$ higher than that previously reported for single crystal. Besides, our results establish that there are only features, which however, differ from those expected for unconventional superconductivity and spin fluctuations reported above.

\section{Experimental details}

A polycrystalline sample of $\mathrm{Mo}_{3} \mathrm{Sb}_{7}$ was prepared from Mo and Sb powders (purity $99.95 \%$ from Alfa Aesar) by solid state reaction. The components were mixed in a stoichiometric ratio pressed into a pellet and sealed in an evacuated quartz tube. The ampoule was then annealed at $750^{\circ} \mathrm{C}$ for one week. The purity, homogeneity and crystal structure of the sample were checked by X-ray diffraction. Lattice parameter of the investigated sample was refined in the Rietveld analysis using the WinPLOTR program [5]. The purity of the sample was also checked by a scanning electron microscope Phillips 515 and an energy dispersive X-ray spectrometer PV9800.

Magnetization measurements were performed using a SQUID magnetometer (MPMS-5T, Quantum Design) in magnetic fields up to $5 \mathrm{~T}$ and in the temperature range $1.8-5 \mathrm{~K}$. Electrical resistivity was measured by an ac four-probe method in the temperature range $0.4-75 \mathrm{~K}$ and in magnetic fields up to $4 \mathrm{~T}$. The measured sample has a dimension $1 \times 1 \times 5 \mathrm{~mm}^{3}$. The Au wires were assembled onto the sample using silver paste. The applied current was $5 \mathrm{~mA}$. Specific heat measurement was performed on a solid $30 \mathrm{mg}$ piece using the thermal relaxation method in the temperature range $0.4-10 \mathrm{~K}$ and in magnetic fields up to $2 \mathrm{~T}$. The piece was glued with a small amount $(0.1 \mathrm{mg})$ of Apizon $\mathrm{N}$ grease onto a sapphire platform. The electrical resistivity and specific heat were collected using a PPMS system (Quantum Design).

\section{Results and discussion}

\subsection{Sample characterization}

Figure 1 shows the experimental powder X-ray diffraction patterns of $\mathrm{Mo}_{3} \mathrm{Sb}_{7}$ as well as the profile fit and difference patterns in the Rietveld analysis. It can be seen that the observed Bragg reflections were indexed on the base of the cubic $\operatorname{Ir}_{3} \mathrm{Ge}_{7}$-type structure (space group $\operatorname{Im} \overline{3} m$ ) [6]. In the refinements, we assumed that Mo atoms occupy the $12 \mathrm{e}(x=0.342)$ and the antimony distribute on the $12 \mathrm{~d}$ and $16 \mathrm{f}(x=0.162)$ sites, respectively. The refinements of the X-ray 


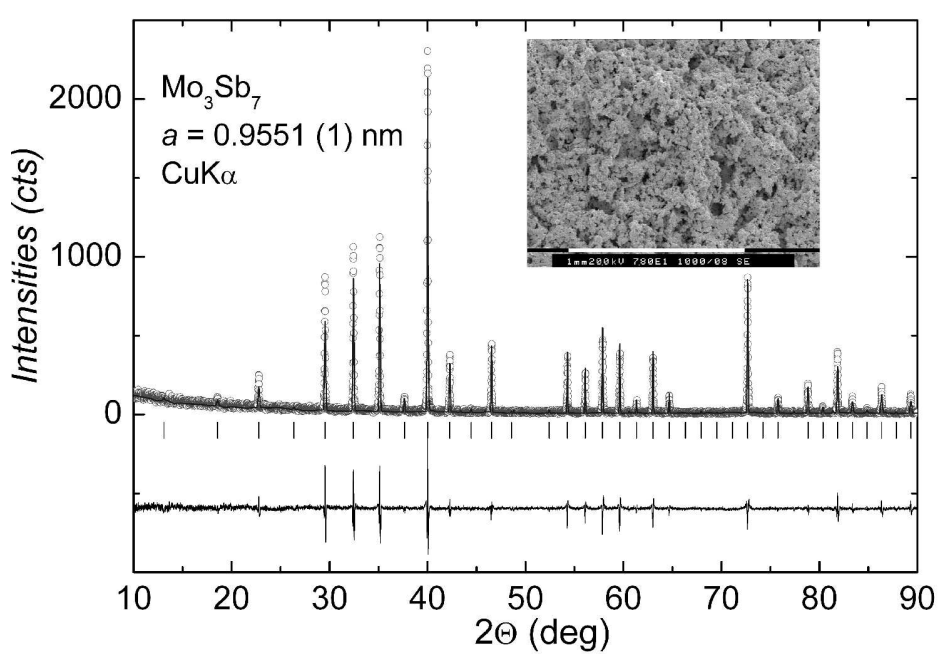

Fig. 1. Powder X-ray diffraction pattern of $\mathrm{Mo}_{3} \mathrm{Sb}_{7}$. The observed (open circles) and calculated (solid line) profiles are shown on the top. The vertical marks in the middle are calculated positions of the Bragg peaks. The line in bottom of the plot is the difference between calculated and observed intensities. The SEM image of the typical samples is shown in the inset.

intensities yield lattice parameter $a=0.9551(1) \mathrm{nm}$, in good agreement with that previously reported by Dashjav et al. for a single crystal $(0.9559 \mathrm{~nm})$ [7].

The microanalysis was performed by collecting several energy dispersive X-ray (EDX) spectra at various locations across the surface of a sintered pellet. The compositions determined by EDX Mo: $29.2 \pm 3$ at. $\%$ and Sb: $70.8 \pm 3$ at. $\%$ are close to the ideal composition $\mathrm{Mo:Sb}=3: 7$. No impurity phase within the experimental error is observed in the EDX spectra. The scanning electron microscopy (SEM) image of the typical samples is shown in the inset of Fig. 1.

\subsection{Bulk physical properties}

In Fig. 2a we show the susceptibility $\chi_{v}$, defined as the magnetization divided by the applied field, $M / H$, as a function of temperature for $T<2.4 \mathrm{~K}$. It can be seen that the onset of diamagnetism due to the superconductivity appears at $2.28 \mathrm{~K}$. The volume fraction of superconductivity at $1.78 \mathrm{~K}$ is estimated to be about $30 \%$ from the field cooling susceptibility at $0.5 \mathrm{mT}$ and $45 \%$ of the perfect shielding from zero-field cooling susceptibility at $10 \mathrm{mT}$. Figure $2 \mathrm{~b}$ represents the magnetization versus magnetic field curve at $1.78 \mathrm{~K}$ in the magnetic field range -0.3 to $0.3 \mathrm{~T}$. The $M-H$ curve displays the typical type-II superconductor. In the low field range, the $M-H$ curve follows a linear dependence and the $\mu_{0} H_{\mathrm{c} 1}$ at $1.78 \mathrm{~K}$, taken as the point where the magnetization starts to deviate from the linear field dependence, can be estimated to be $3.2 \mathrm{mT}$.

The electrical resistivity measurements establish the superconducting transition with $T_{\mathrm{c}}=2.26 \mathrm{~K}$ and the transition width $\Delta T_{\mathrm{c}}=T_{\mathrm{c}}(90 \%)-T_{\mathrm{c}}(10 \%)=0.12 \mathrm{~K}$ 

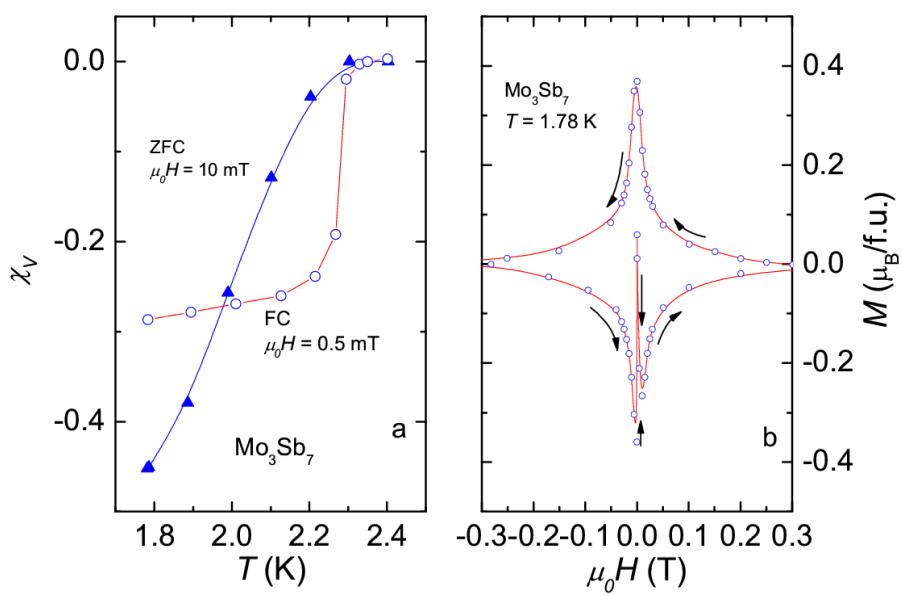

Fig. 2. (a) Temperature dependence of volume susceptibility of $\mathrm{Mo}_{3} \mathrm{Sb}_{7}$ measured at $0.5 \mathrm{mT}$ and $10 \mathrm{mT}$. (b) The field dependence of the magnetization of $\mathrm{Mo}_{3} \mathrm{Sb}_{7}$ at $1.78 \mathrm{~K}$. The arrows indicate the direction of applied magnetic fields.

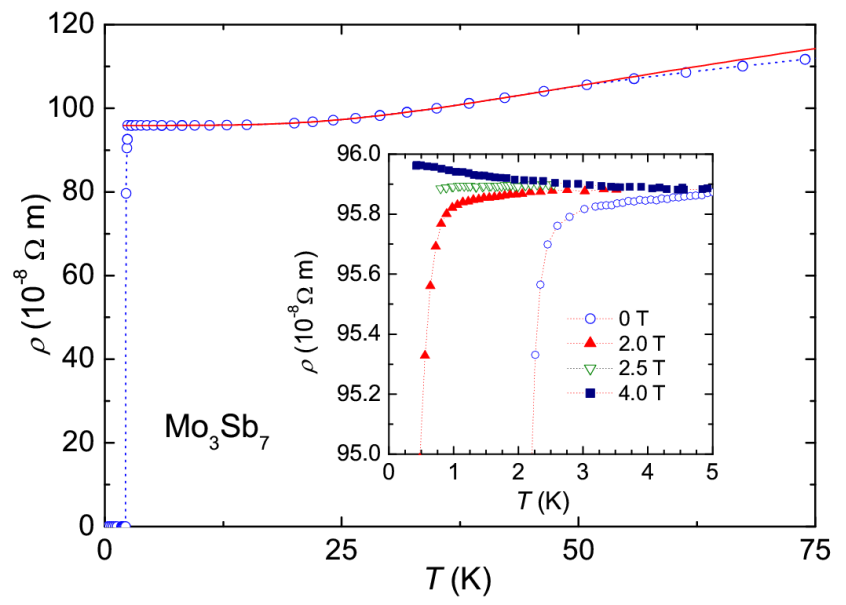

Fig. 3. Temperature dependence of the the electrical resistivity below $75 \mathrm{~K}$. The inset shows low-temperature resistivity at several magnetic fields up to $4 \mathrm{~T}$. The solid line is the fit (see text) and dashed lines a guide to the eye.

(Fig. 3). This fact together with the magnetic data support a high degree of homogeneous superconductivity of the investigated sample. Inspecting the $\rho(T)$-curve we recognize that the temperature dependence of the resistivity up to $40 \mathrm{~K}$ does not follow either $T^{2}$ or $T^{5}$-dependence. Instead, the resistivity can be fitted to the equation: $\rho(T)=\rho_{0}+c T+d \exp \left(-\Delta_{\rho} / k_{\mathrm{B}} T\right)$, with $\rho_{0}=96 \times 10^{-8} \Omega \mathrm{m}$, $c=0.022 \times 10^{-8} \Omega \mathrm{m} / \mathrm{K}, d=75 \times 10^{-8} \Omega \mathrm{m}$ and $\Delta_{\rho} / k_{\mathrm{B}}=110$ (2) K. Due to the porosity of the studied sample, the absolute value of the resistivity in the normal 
state should be interpreted with caution. Let us note that the above equation was successfully used by Woodward and Cody [8] for $\mathrm{Nb}_{3} \mathrm{Sn}$, which is a superconductor with significant electron-phonon coupling strength. The presence of the $\exp \left(-\Delta_{\rho} / k_{\mathrm{B}} T\right)$ term may account for a rapid increase in the resistivity with increasing temperature, compared to the $T^{2}$ or $T^{5}$ dependence usually observed in metals.

An application of magnetic fields shifts the superconducting phase transition to lower temperatures. At $0.4 \mathrm{~K}$, the vanishing of superconductivity is observed at $\mu_{0} H_{\mathrm{c} 2}=2 \mathrm{~T}$, which is in good agreement with the specific heat data (see below). An important result emerging from our data is the observation of an upturn in the resistivity measured in magnetic fields. The observed positive magnetoresistance (MR) may preclude an interpretation in terms of spin fluctuations, for which the $\mathrm{MR}$ is usually negative [9].
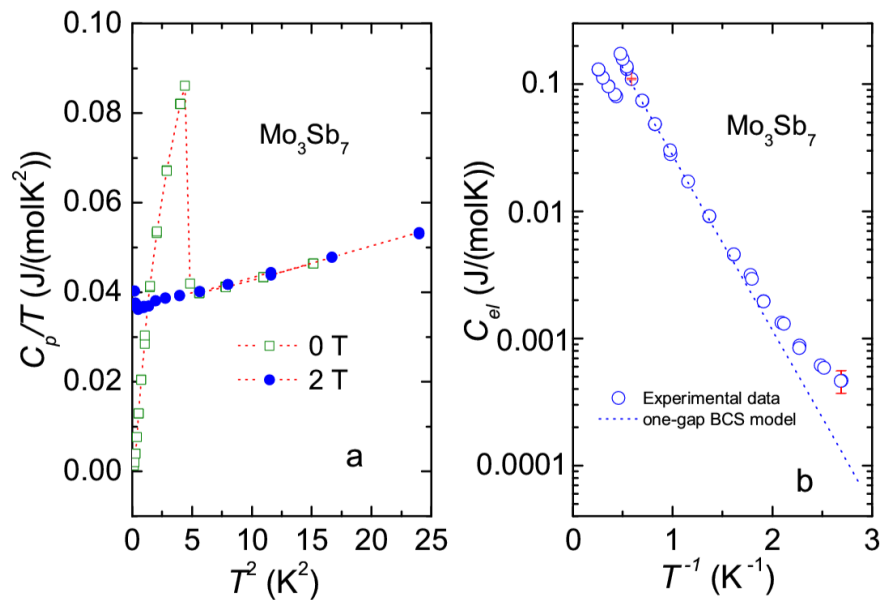

Fig. 4. (a) The temperature dependence of the specific heat divided by temperature at 0 and $2 \mathrm{~T}$. (b) $\ln \left(C_{\mathrm{el}}\right)$ as a function of the inverse temperature $1 / T$. Error bars at $0.4 \mathrm{~K}$, as large as $\pm 20 \%$, and at $1.7 \mathrm{~K}$ of $\pm 2 \%$, were taken from [21]. The dashed line represents the BCS theoretical curve of the formula: $C_{\mathrm{el}} / \gamma T_{\mathrm{c}}=8.5 \exp \left(-1.44 T_{\mathrm{c}} / T\right)$.

Figure $4 \mathrm{a}$ shows the specific heat data collected at 0 and $2 \mathrm{~T}$ in the form of $C_{p} / T$ vs. $T^{2}$. The zero-field data exhibit the distinct superconducting phase transition at $\approx 2.3 \mathrm{~K}$, confirming that the superconductivity in the studied sample is of bulk nature. The normal-state specific heat of $\mathrm{Mo}_{3} \mathrm{Sb}_{7}$ can be fitted to the formula

$$
C_{p}=\gamma_{n} T+\beta T^{3},
$$

where the first term denotes the electronic contribution and the second the lattice contribution to the total specific heat. The values $\gamma_{n}$ and $\beta$ have been determined from the fitting of the data for $T>2.5 \mathrm{~K}$. We obtained $\gamma_{n}=34.5(1) \mathrm{mJ} /\left(\mathrm{mol} \mathrm{K}^{2}\right)$ 
and $\beta=0.85(5) \mathrm{mJ} /\left(\mathrm{mol} \mathrm{K}^{4}\right)$. From the latter value we may estimate the Debye temperature by using the relation

$$
\Theta_{\mathrm{D}}=\left(\frac{12 \pi^{4} n R}{5 \beta}\right)^{1 / 3}
$$

with $n=10$ and $R$ is the gas constant. The value of the Debye temperature is found to be $283(3) \mathrm{K}$. From the specific heat jump $\Delta C_{p}\left(T_{\mathrm{c}}\right)$ at $T_{\mathrm{c}}$ and $\gamma_{n}$ value we may estimate the ratio $\Delta C_{p}\left(T_{\mathrm{c}}\right) / \gamma_{n} T_{\mathrm{c}}=1.56$, which is larger than the BCS weak-coupling value of 1.43 .

It is noted that the value of $\gamma_{n}$ for $\mathrm{Mo}_{3} \mathrm{Sb}_{7}$ is relatively large $\left(3.45 \mathrm{~mJ} /\left(\mathrm{g}\right.\right.$ at. $\left.\left.\mathrm{K}^{2}\right)\right)$ as one compares to those of the high- $T_{\mathrm{c}}$ (HTc) superconductor $\mathrm{YBa}_{2} \mathrm{Cu}_{3} \mathrm{O}_{7}\left(1.5 \mathrm{~mJ} / \mathrm{g}\right.$ at $\left.\mathrm{K}^{2}[10]\right)$ and double gap superconductor $\mathrm{MgB}_{2}$ $\left(0.8 \mathrm{~mJ} / \mathrm{g}\right.$ at $\left.\mathrm{K}^{2}[11]\right)$. In similar manner as for the A15 superconductors [12], the observed enhanced $\gamma$ value in $\mathrm{Mo}_{3} \mathrm{Sb}_{7}$ can be explained due to a substantial contribution of the $4 d$-electrons of the Mo atoms to the DOS at the Fermi level.

In order to evaluate the electron-phonon coupling constant $\bar{\lambda}_{\mathrm{e}-\mathrm{ph}}$, we use the modified McMillan expression [13, 14]:

$$
k_{\mathrm{B}} T_{\mathrm{c}}=\frac{\hbar \omega_{\mathrm{log}}}{1.2} \exp \left(-\frac{1.04\left(1+\bar{\lambda}_{\mathrm{e}-\mathrm{ph}}\right)}{\bar{\lambda}_{\mathrm{e}-\mathrm{ph}}-\mu^{*}\left(1+0.62 \bar{\lambda}_{\mathrm{e}-\mathrm{ph}}\right)}\right) .
$$

$\omega_{\log }$ can be approximated as $\omega_{\log }=0.8 \omega_{\mathrm{g}}$ and $\omega_{\mathrm{g}}=0.7 k_{\mathrm{B}} \Theta_{\mathrm{D}} / \hbar[15] . \mu^{*}$ is the Coulomb repulsion between electrons [16] and usually amounts to 0.1-0.2. Putting $\mu^{*}=0.15$ into Eq. (3) we get $\bar{\lambda}_{\mathrm{e}-\mathrm{ph}}=0.6$. The relatively large values of $\bar{\lambda}_{\mathrm{e}-\mathrm{ph}}$ and $\Delta C_{p}\left(T_{\mathrm{c}}\right) / \gamma_{n} T_{\mathrm{c}}$ suggest an intermediate strong coupling strength in $\mathrm{Mo}_{3} \mathrm{Sb}_{7}$.

A magnetic field of $2 \mathrm{~T}$ shifts the superconducting transition down to $0.4 \mathrm{~K}$, but remains the constant value of $\gamma_{n}$. Thus, in addition to the fact that the $C_{p}$ does not vary as a function of $T^{3} \ln T$, there also is a lack of field dependence of the $\gamma_{n}$. Since, an eventual existence of these two features is finger pointing spin fluctuations $[17,18]$, our observation of the $C_{p}(T)$ behavior makes a question about role of spin fluctuations proposed previously.

The electronic heat capacity is determined via the difference $C_{\mathrm{el}}=C_{p}-\beta T^{3}$ and is shown in Fig. $4 \mathrm{~b}$ in the form of semi-logarithmic graph $\ln \left(C_{\mathrm{el}}\right)$ vs. $1 / T$. The figure displays also the theoretical curve predicted by the BCS theory $C_{\mathrm{el}} / \gamma T_{\mathrm{c}}=$ $8.5 \exp \left(-1.44 T_{\mathrm{c}} / T\right)$ for $2.5<T_{\mathrm{c}} / T<6[19]$. A comparison of the experimental with the theoretical curve points out that the electronic specific heat data of $\mathrm{Mo}_{3} \mathrm{Sb}_{7}$ deviate from the isotropic BCS theory for weak-coupling superconductors. Instead, the data may be described by a model assuming the presence of two superconducting BCS-like temperature dependence gaps [20]. In the model, the electronic specific heat below $T_{\mathrm{c}}$ was considered as the sum of the contributions of two bands characterized by respective gap widths $\Delta_{1}$ and $\Delta_{2}$, and by partial Sommerfeld ratio $\gamma_{1}$ and $\gamma_{2}$, where $\gamma_{n}=\gamma_{1}+\gamma_{2}$. The gap widths have been found to be $2 \Delta_{1} / k_{\mathrm{B}} T_{\mathrm{c}}=4.0$ and $2 \Delta_{2} / k_{\mathrm{B}} T_{\mathrm{c}}=2.5$, and relative mole weights of the mole electronic heat coefficients to be $\gamma_{1} / \gamma_{n}=0.7$ and $\gamma_{2} / \gamma_{n}=0.3$. 


\section{Conclusion remarks}

We have synthesized polycrystalline sample of the superconductor $\mathrm{Mo}_{3} \mathrm{Sb}_{7}$ and characterized by means of X-ray diffraction and dispersive spectroscopy. We have determined some basic physical properties of $\mathrm{Mo}_{3} \mathrm{Sb}_{7}$ in superconducting state. From the experimental data we conclude that the investigated compound with the Mo $4 d$-electrons enhancing density of states, undergoes superconducting state at $2.25(0.05) \mathrm{K}$. The studied compound may be classified to the class of type-II superconductor of a moderate BCS-like coupling strength. Our experimental data do not give evidence for either unconventional superconductivity or spin fluctuations, thus in order to determine proper mechanism for electron pairing in $\mathrm{Mo}_{3} \mathrm{Sb}_{7}$ further investigations are required.

\section{Acknowledgments}

We would like to thank E. Bukowska for kind performing X-ray diffraction and K. Nierzewski for SEM/EDX measurements. The work at ILT \& SR was supported by the Ministry of Science and Higher Education under grant No. N202 $08231 / 0449$.

\section{References}

[1] Z. Bukowski, D. Badurski, J. Stẹpień-Damm, R. Troć, Solid State Commun. 123, 283 (2002).

[2] V.M. Dmitriev, L.F. Rybaltchenko, L.A. Ishchenko, E.V. Khristenko, Z. Bukowski, R. Troć, Supercond. Sci. Technol. 19, 573 (2006).

[3] V.M. Dmitriev, L.F. Rybaltchenko, E.V. Khristenko, L.A. Ishchenko, Z. Bukowski, R. Troć, Fiz. Nizk. Temp. 33, 399 (2007).

[4] C. Candolfi, B. Lenoir, A. Dauscher, C. Bellouard, J. Hejtmanek, E. Santava, J. Tobola, Phys. Rev. Lett. 99, 037006 (2007).

[5] T. Roisnel, J. Rodriguez-Carvajal, Program WinPLOTR, http://www- llb.cea.fr/winplotr/winplotr.htm.

[6] P. Jensen, A. Kjekshus, T. Skansen, Acta Chem. Scand. 20, 403 (1966).

[7] E. Dashjav, A. Szczepenowska, H. Kleinke, J. Mater. Chem. 12, 345 (2002).

[8] D.W. Woodward, G.D. Cody, Phys. Rev. 136, A166 (1964).

[9] H. Yamada, S. Takada, Prog. Theor. Phys. 48, 1828 (1972).

[10] A. Junod, in: Studies of High Temperature Superconductors, Ed. A. Narlikar, Nova Science Publ., Commack, New York 1996, p. 191.

[11] Y. Wang, F. Bouquet, I. Sheikin, P. Toulemonde, B. Revaz, M. Eisterer, H. Weber, J. Hinderer, A. Junod, J. Phys., Condens. Matter 15, 883 (2003).

[12] T. Jarlborg, A. Junod, M. Peter, Phys. Rev. B 27, 1558 (1983).

[13] W.L. McMillan, Phys. Rev. 157, 331 (1968).

[14] P.B. Allen, R.C. Dynes, Phys. Rev. B 12, 905 (1975).

[15] B.M. Klein, W.E. Pickett, D.A. Papaconstantopoulos, L.L. Boyer, Phys. Rev. B 27, 6721 (1983). 
[16] P. Morel, P.W. Anderson, Phys. Rev. 125, 1263 (1962).

[17] W.F. Brinkman, S. Engelsberg, Phys. Rev. 169, 417 (1968).

[18] M.T. Béal-Monod, S.-K. Ma, D.R. Fredkin, Phys. Rev. Lett. 20, 929 (1968).

[19] E.S.R. Gopal, Specific Heat at Low Temperatures, Plenum Press, New York 1966.

[20] V.H. Tran, W. Miiller, Z. Bukowski, ArXiv:0803.2948; Phys. Rev. Lett. 100, 137004 (2008)

[21] R.A. Marriott, M. Stancescu, C.A. Kennedy, M.A. White, Rev. Sci. Instrum. 77, 96108 (2006). 